\title{
CUSTOS DE BEM-ESTAR DA INFLAÇÃO NO BRASIL PÓS-REAL
}

\author{
Sidney Martins Caetano * \\ Geraldo Edmundo Silva Júnior ${ }^{\dagger}$ \\ José Ronaldo de Castro Souza Júnior ‡
}

\begin{abstract}
Resumo
Este trabalho investiga o comportamento da demanda por moeda no Brasil de forma a derivar os custos de bem-estar da inflação pós-Plano Real. Os resultados, para um período de dezessete anos, corroboram a forma funcional log-log que mostra significativos ganhos de bem-estar relativos aos custos sociais anteriores ao Plano Real. Para uma inflação de aproximadamente $4,5 \%$ a.a., tem-se custo de bem-estar entre 0,15 e $0,20 \%$ da renda. Conclui-se, como esperado, que o país deve direcionar suas políticas para alcançar tanto um patamar de inflação menor quanto para obter um nível de taxa de juros real de estado estacionário de países desenvolvidos.
\end{abstract}

Palavras-chave: Demanda por Moeda; Custos de Bem-Estar da Inflação; Política Monetária.

\begin{abstract}
This work investigates the behavior of money demand in Brazil in order to derive the welfare costs of inflation after the Real Plan. The results, for a period of about seventeen years, corroborated the log-log specification that shows significant gains in welfare cost of inflation. Still, for an inflation of around $4.5 \%$ per year, there is a welfare cost between 0,15 and $0,20 \%$ of income. But, we suggest that the country directs its policies to achieve a much lower level of inflation and to a level of steady state real interest rate of developed countries.
\end{abstract}

Keywords: Money Demand; Welfare Cost of Inflation; Monetary Policy. JEL classification: E31, E41, E52.

DOI: http://dx.doi.org/10.1590/1413-8050/ea440

\footnotetext{
* Universidade Federal de Juiz de Fora - UFJF. Programa de Pós-Graduação em Economia Aplicada. E-mail: sidney.caetano@ufjf.edu.br

† Universidade Federal de São Carlos - UFSCar. Programa de Pós-Graduação em Economia. E-mail: gedmundos@gmail.com

‡ Instituto de Pesquisa Econômica Aplicada — IPEA. E-mail: ronaldo.souza@ipea.gov.br
} 


\section{Introdução}

A inflação no Brasil, no período pós-Plano Real, apresentou patamares inferiores aos dois dígitos. Tal resultado nos conduz à discussão dos custos sociais gerados ao se manter uma inflação baixa, mas ainda positiva, quando comparado à política monetária ótima, proposta por Friedman (1969), que defende uma deflação que leve a uma taxa nominal de juros nula.

$\mathrm{Na}$ literatura tem-se que a inflação apresenta custos e benefícios para uma economia dependendo do seu nível e do patamar associados com a taxa de juro. Em geral, os custos da inflação facilmente identificáveis são aqueles do tipo "sola de sapato" (shoeleather costs), que, segundo Romer (2005), levam os agentes econômicos a deter cada vez menos encaixes em moeda. Além disso, os preços e os salários nominais passam a ser alterados com maior frequência podendo gerar um processo de indexação. A inflação também pode causar distorção no sistema fiscal ao elevar a taxa efetiva de impostos.

Outros custos relacionados a uma inflação estável devem-se ao ajustamento defasado dos preços causando variações nos preços relativos, dificultando relações de longo prazo e a eficiência dos mercados. Os agentes econômicos podem sofrer de "ilusão monetária", aumentando, assim, a probabilidade de ineficiência das decisões de investimento e de poupança. Em relação aos custos de uma inflação variável destaca-se o caso da aquisição de ativos denominados nominalmente, pois variações não antecipadas da inflação redistribuem riqueza.

Dado que a inflação não gera apenas custos, torna-se relevante citar alguns dos seus benefícios potenciais, a saber: se o salário nominal for rígido, principalmente para baixo, os salários reais podem ser facilmente ajustados, se a inflação for elevada; optando por uma taxa real de juros negativa, os policymakers podem gerar uma política macroeconômica mais flexível; e, em uma economia com taxa média de inflação maior, têm-se mais oportunidades de se usar a política monetária para combater recessão. Uma inflação abaixo de determinado patamar pode ser prejudicial à economia, razão por que alguma inflação (baixa a moderada) poderá ser desejável. Por isso, a importância e, ao mesmo tempo, complexidade em definir uma meta de inflação adequada para as economias em que vigoram o regime de metas para inflação.

A despeito de não se enfrentar no Brasil um problema de inflação alta e crônica há mais de uma década e meia, mas diante dos custos de bem-estar associados a esse fenômeno, quando positivo, torna-se relevante estimar e analisar o comportamento da demanda por moeda e dos custos sociais da inflação, em termos de renda, para a economia brasileira.

Inúmeras considerações teóricas e empíricas foram sendo desenvolvidas na literatura com desdobramentos importantes sobre o tema. A contribuição seminal é devida a Bailey $(1956)^{1}$ que aponta um custo de bem-estar advindo do imposto inflacionário do governo gerado pela desvalorização da moeda via emissão monetária. Em seu estudo, o elemento crucial é a separação entre os efeitos inflacionários e o custo de bem-estar associado com o efeito encaixesreais. As contribuições de Cagan (1956) e, posteriormente, de Meltzer (1963), objetivaram a determinação da forma funcional da demanda por moeda com Cagan (1956) estabelecendo a forma semi-log e Meltzer (1963) a forma

\footnotetext{
${ }^{1}$ Harberger (1971) se constitui em uma alternativa à medida de Bailey quando ativos de menor liquidez são considerados.
} 
$\log$-log. Especificações que geram distintos custos de bem-estar da inflação, portanto, relevantes para sua determinação.

Com a hipótese da instituição de uma política monetária ótima, com taxa de inflação nula ou estabilidade de preços, para que ocorra uma taxa nominal de juros baixa e próxima de zero, surge a necessidade do tratamento empírico do tema para a mensuração do custo de bem-estar da inflação. Nesse sentido, considerações decorrentes das especificações das formas semi-log e log-log foram propostas na literatura empírica, sendo que a estrutura semi-log descreve bem ambientes de elevada inflação, enquanto a log-log seria robusta para estruturas de baixa inflação, ver Yavari \& Serletis (2011). Conforme Serletis \& Yavari (2004), a forma funcional log-log ajusta-se melhor a dados que não incluem regiões de hiperinflação ou taxas de juros quase nulas.

Lucas (2000) quantificou as especificações de Cagan e de Meltzer para um período longo de tempo, correspondendo aos dados anuais para o intervalo entre 1900 -1994, para o qual o autor concluiu pela superioridade da forma log-log. Recentemente, Ireland (2009) utilizou uma abordagem econométrica mais sofisticada ao utilizar testes de raízes unitárias e de cointegração, desenvolvendo inferências robustas para um período mais curto, correspondendo aos dados trimestrais de $1980-I$ à 2006-IV. Seus resultados apontaram para uma especificação semi-log, portanto, diferente da obtida por Lucas. Consequentemente, gerou-se uma política monetária diferente daquela proposta por Friedman (1969), com custos de bem-estar inferiores aos estimados por Lucas (2000).

É notório que, mesmo em modelos microfundamentados, as implicações de bem-estar estariam vinculadas às propriedades de longo prazo da demanda por moeda, o que torna a análise de Ireland (2009) importante por ajustar a forma funcional aos dados observados, permitindo melhores calibrações dos modelos quando necessário. Serletis \& Yavari (2004) destacam que o conhecimento acerca da elasticidade-juros da demanda de moeda é crucial na avaliação dos custos de bem-estar da inflação.

Dessa forma, o presente estudo contribui com a literatura empírica brasileira sobre demanda por moeda para o estudo dos custos de bem-estar da inflação utilizando a robustez do procedimento de Ireland (2009), considerando a discussão sobre as especificações log-log e semi-log para demanda por moeda, que mesmo diante de abordagens de equilíbrio geral ${ }^{2}$, que se diz mais ampla, tem recebido muita atenção. Já os custos de bem-estar da inflação foram obtidos tanto por meio da aproximação de Bailey quanto da aproximação de Simonsen \& Cysne (2007). O período abordado envolve dados trimestrais brasileiros de 1995 - 2011, o que permitiu inferir sobre a elasticidade da demanda de moeda relativa a juros e renda, bem como aos ganhos de bem-estar da política de estabilização de preços imposta ao país a partir do Plano Real.

Para tanto, o presente trabalho segue assim distribuído: a presente introdução que constitui a seção 1 ; a seção 2 apresenta referêncial teórico da teoria de custos de bem-estar da inflação e uma breve discussão da literatura empírica da demanda de moeda no Brasil; a seção 3 apresenta os dados, as estimativas e sua discussão; a seção 4 estima os custos de bem-estar da inflação; e, a seção 5 finaliza com as considerações finais.

\footnotetext{
${ }^{2}$ Ver, por exemplo, Yoshino (2002).
} 


\section{A Teoria de Custos de Bem-Estar da Inflação e os Estudos Empíricos sobre Demanda de Moeda no Brasil}

Uma característica importante da teoria de custos de bem-estar da inflação é que a mesma se circunscreve a duas estruturas de demanda por moedas fechadas, a estrutura proposta por Cagan (semi-log) e a estrutura proposta por Meltzer (log-log). A mensuração do custo de bem-estar decorre de duas abordagens devidas a Bailey (1956) e Harberger (1971), respectivamente.

\subsection{A teoria de custos de bem-estar da inflação}

Originalmente, o enfoque teórico da análise de custos de bem-estar, da inflação foi iniciado por Bailey (1956). Em sua estrutura teórica, o autor utiliza a abordagem do excedente do consumidor para mensurar os custos de bemestar, integrando a função de demanda por moeda, com o limite inferior que corresponde a uma taxa de juros nula e superior a uma taxa de juros positiva, com o resultado descontado pela receita de senhoriagem.

Friedman (1969), ao sugerir uma política monetária ótima com taxa de inflação nula, instaurou o debate acerca dos custos de uma inflação positiva e os seus efeitos sobre a transferência de renda do setor privado para o governo e da perda de bem-estar em relação aos custos associados à inflação.

Diante de tais questionamentos, os custos e os benefícios de uma taxa de inflação positiva, mesmo em baixos níveis, passaram a ser discutidos tanto na literatura teórica quanto na empírica. Em síntese, a preocupação central na literatura empírica instaurada foi estimar o ganho potencial em termos de bem-estar de se reduzir os patamares inflacionários e de taxas de juros a níveis compatíveis com uma política monetária ótima.

Então, três décadas mais tarde, após os questionamentos de Friedman (1969), a literatura sobre os custos sociais da inflação ganhou novo reforço com o trabalho de Lucas (2000), onde o autor leva a discussão para o nível empírico ao estimar os custos de bem-estar da inflação. Para tanto, as formas funcionais propostas por Cagan (1956), semi-log, e por Meltzer (1963), log$\log$, foram consideradas, sendo esta última preferida por ajustar-se melhor aos dados americanos.

Nesse ínterim surgiram desdobramentos teóricos e empíricos quanto ao agregado adequado e ao tipo de especificação para a demanda de moeda em que os custos sociais se dariam. Uma contribuição dada por Simonsen \& Cysne (2001) foi incorporar no cômputo dos custos de bem-estar da inflação os depósitos remunerados. Com tal estrutura, portanto, tornou-se possível a análise teórica do tema com a ampliação do conceito de moeda, desta vez não mais restrita aos encaixes em moeda, ou papel moeda em poder do público mais depósitos à vista não remunerados.

A dificuldade de desenvolvimento teórico foi estendida ao conceito do agregado monetário, pois a agregação por soma simples conduziria a um viés na aferição dos custos e na interpretação dos resultados em função da alocação de portfólio dos agentes privados. Em uma segunda contribuição, Cysne (2003) incorporou o conceito de "índice de divisa" como estrutura adequada ao possibilitar a captura das inovações financeiras e, também, o estudo do tema em ambientes inflacionários, nos quais ocorre a substituição entre ativos financeiros líquidos e ativos financeiros menos líquidos que rendem juros. Em uma terceira contribuição, que se refere à discussão sobre a estrutura de 
equilíbrio da função de demanda por moeda considerada, Cysne (2005) usou uma forma fechada de equilíbrio geral que permitiu o cálculo da diferença dos custos entre as estruturas de equilíbrio parcial e geral. Essa abordagem foi rediscutida por Cysne (2009), quando o autor analisou o viés de outras medidas presentes na literatura e aproximou a medida de Bailey a uma medida de equilíbrio geral.

O viés da agregação foi reconsiderado em Cysne \& Turchick (2010) em que as condições necessárias e suficientes para a estimação da medida foram especificadas e, ainda, a magnitude do viés em forma fechada foi derivada.

Embora o avanço tenha ocorrido na literatura teórica, algumas contribuições foram expressivas no que concerne à definição e à quantificação da especificação da função de demanda de moeda que leva aos custos de bem-estar. Por exemplo, o tema foi empiricamente tratado por Lucas (2000) e Ireland (2009), que diferem entre os períodos analisados e procedimentos utilizados. Lucas, baseando-se em Bailey (1956) e Friedman (1969), aborda a questão diretamente. Sua análise justapôs duas especificações concorrentes para a demanda por moeda. Uma primeira, inspirada em Meltzer (1963), relaciona o logaritmo natural de $\mathrm{m}$, a razão entre saldos monetários nominais e renda nominal, com relação ao logaritmo natural da taxa nominal de juros de curto prazo $r$, ou seja:

$$
\ln (m)=\ln (A)-\eta \ln (r) .
$$

Na especificação (1), $A>0$ é uma constante positiva e $\eta>0$ mensura o valor absoluto da elasticidade-juros da moeda. A segunda, baseada em Cagan (1956), relaciona o mesmo logaritmo natural de $m$ com relação à taxa de juros de curto prazo em nível, $r$, tal como:

$$
\ln (m)=\ln (B)-\xi r,
$$

onde $B>0$ é uma constante positiva e $\xi>0$ representa o valor absoluto da semi-elasticidade-juros da moeda.

A abordagem de Bailey (1956) utiliza a ideia do excedente do consumidor para mensurar o custo de bem-estar ao integrar a curva de demanda por moeda em relação à taxa de juros no intervalo de zero a $r, \operatorname{com} r>0$ para, então, subtrair este valor da receita de senhoriagem $(\mathrm{rm})$ isolando, assim, o custo social. Ou seja,

$$
\begin{gathered}
w(r)=\int_{0}^{r} A \tau^{-\eta} d \tau-r A r^{-\eta}=\left.\frac{A \tau^{1-\eta}}{1-\eta}\right|_{0} ^{r}-r A r^{-\eta}=\frac{A r^{1-\eta}}{1-\eta}-r A r^{-\eta} \\
w(r)=\frac{(1-1+\eta) A r^{1-\eta}}{1-\eta}=\frac{\eta}{1-\eta} A r^{1-\eta} .
\end{gathered}
$$

Se $w(r)$ representa a medida do custo de bem-estar, então, quando a demanda por moeda toma a forma log-log, equação (1), é possível expressá-la como uma função de $r$, ou seja,

$$
w(r)=A\left(\frac{\eta}{1-\eta}\right) r^{1-\eta}
$$

No caso em que a demanda por moeda toma a forma semi-log, equação (2), a expressão torna-se: 


$$
w(r)=\frac{B}{\xi}\left[1-(1+\xi r) e^{-\xi r}\right] .
$$

Se, como pressuposto por Lucas (2000), a taxa real de juros no estado estacionário for equivalente a 3\%, de modo que $r=0,03$ prevalece no âmbito de uma política de inflação nula ou de estabilidade de preços, então, (3) e (4) resultam que esta política custa à economia americana o equivalente a $0,9 \%$ da renda quando a demanda por moeda é log-log, mas apenas $0,1 \%$ da renda quando a demanda por moeda tem a forma semi-log. O autor escolhe diante dos ajustes frente aos dados anuais americanos $(1900-1994)$ um valor de $\eta=0,5$ e $\xi=7$, para em seguida calcular o valor de $A$ e $B$.

Ireland (2009) também investigou o comportamento da demanda por moeda e os custos de bem-estar da inflação americana, mas utilizando testes de raízes unitárias de Phillips-Perron, teste de cointegração de Phillips-Ouliaris e OLS dinâmico de Stock-Watson para um período diferente de tempo. Supôs uma taxa real de juros anual de estado estacionário também equivalente a $3 \%$ (ou $r=0,03$ ), o que corresponde a uma taxa de inflação anual nula; enquanto uma taxa de inflação de $2 \%$ e $10 \%$ implicam em uma taxa nominal de juros de 5 e 13\%, respectivamente. Os coeficientes das regressões estimadas permitiram encontrar custos de bem-estar de menos de 0,015\% da renda quando a taxa de inflação é nula, de menos de $0,04 \%$ da renda se a inflação for de $2 \%$, e a menos de $0,25 \%$ da renda com $10 \%$ de inflação. Estimativas de custos de bem-estar que estão muito abaixo das obtidas por Lucas (2000).

Simonsen \& Cysne (2007) apresentam um resultado derivado originalmente em Simonsen \& Cysne (2001), que representa uma solução fechada para uma equação diferencial que aproxima a derivação de Lucas. Essa aproximação situa-se mais próxima da solução da equação original do que a aproximação dada pela fórmula de Bailey. Usando também o caso de demanda por moeda $\log$-log, equação (1), os autores chegam à seguinte expressão:

$$
w(r)=\frac{\eta}{1-\eta} \ln \left(1+A r^{1-\eta}\right),
$$

que seria uma melhor aproximação do custo de bem-estar da inflação do que a aproximação de Bailey, equação (3).

Especificamente para a economia brasileira, pode-se destacar outros trabalhos sobre o tema, diferentemente estruturado, tais como Dias (2000), Yoshino (2002) e Rossi (2008). Tomando-se como pontos essenciais a estrutura teórica, a abordagem empírica e os resultados, pode-se inferir que Dias (2000) considerou as implicações da inflação sobre bem-estar social e a senhoriagem. Para tanto, a autora discute o conceito de senhoriagem e considera uma função de utilidade com o lazer dependendo tanto do consumo quanto da dotação de encaixes reais. Das condições de primeira ordem do problema de otimização obtém-se a demanda de moeda que juntamente com a utilidade no estado estacionário permite a análise dos custos de bem-estar por meio da função de perda de bem-estar social.

Yoshino (2002) procura derivar os custos de bem-estar da inflação decorrentes da inflação antecipada. Dependendo do nível de aversão ao risco relativo e da equação parametrizada para o consumo antecipado de bens, seus resultados para os custos de bem-estar variam de 16 a 32\% do PIB no ano de 1990 quando a inflação anual superou a marca de 2590\%. Resultado diferente aos obtidos por Pastore (1995) apud Yoshino (2002) que variam entre 
0,1 e $0,45 \%$. O autor duvida desse último resultado, ao questionar como esse valor pode ser inferior ao apresentado em Lucas (2000), 0,9\% do PIB da economia americana. Para ele, o problema está no uso apenas do triângulo de Bailey sob a demanda de moeda por meio da base monetária. Nesse sentido, propôs olhar também para o triângulo de Harberger. O autor insere o conceito de overbanking que tem a ver com a demanda dos agentes econômicos por produtos bancários ao buscarem economizar tempo de transação.

Rossi (2008) utiliza uma abordagem de equilíbrio geral a la Sidrauski, inserindo aspectos ditos inexplorados na literatura brasileira. Especificamente, o autor investiga o papel da inflação no bem-estar do indivíduo, baseando-se na proposta de que a tributação incidiria sobre os valores nominais e não sobre os valores reais dos rendimentos dos indivíduos. Os resultados surpreendem o autor por não destoarem muito de outros resultados anteriores da literatura, esperava-se certa diferença devido à forma de cálculo dos custos de bem-estar da inflação. Embora as contribuições tenham sido estabelecidas, inclusive em período mais recente, observa-se que não existe consenso quanto ao cálculo nem quanto aos procedimentos metodológicos para a obtenção de resultados concernentes ao tema dentro da literatura.

Assim, diante das contribuições e, baseando-se nas equações (1) a (5), tornase relevante apresentar novos resultados empíricos para a curva de demanda por moeda e os custos de bem-estar da inflação dentro do período pós-Plano Real, conforme faz Ireland (2009).

\subsection{Os estudos sobre demanda de moeda no Brasil}

Especificamente, a literatura empírica da demanda por moeda no Brasil envolve considerações importantes em análises que cobrem um período de mais de meio século. Algumas perspectivas da literatura merecem destaque, a saber: (i) os estudos destinaram-se a análise do comportamento da demanda por moeda para se justificar a conduta da política monetária; (ii) a falta de efetividade ou de instrumentalidade da política monetária em conjunturas de inflação crescente; e, (iii) como a variável demanda por moeda se comporta diante das mudanças nos regimes de política monetária a que foi submetida a economia brasileira.

É possível, ainda, dividir a análise em dois períodos distintos. São eles:

1. O período que antecedeu a aceleração inflacionária combatida de maneira heterodoxa, que compreendeu o período anterior aos planos heterodoxos até o ano de 1986: neste foram identificados o problema da forma funcional e a discussão acerca da ampliação ou não do agregado representativo da demanda por encaixes. Foram destacados os trabalhos de Pastore (1969), Silveira (1973), Contador (1974), Barbosa (1978), Prado (1978), Cardoso (1981), Cysne (1985), Rossi (1988), Triches (1992), Barbosa (1993) e Nakane (1994). Nota-se que Barbosa (1978) resenhou os trabalhos de Pastore (1969), Silveira (1973) e Contador (1974) e outros não contemplados nesta revisão. Ainda, os estudos de Cardoso (1981), Cysne (1985) e Rossi (1988) dispuseram três aspectos básicos da literatura empírica: a inclusão do argumento inflação na equação, a ampliação do conceito de moeda e a discussão sobre a instabilidade da demanda por moeda no Brasil em contextos de inflação elevada; 
2. O período pós-planos heterodoxos: são destacados os problemas essenciais da aceleração da inflação e a necessidade de especificação de uma forma funcional adequada ao período. Os trabalhos de Triches (1992), Barbosa (1993), Nakane (1994), Rossi (1994), Barbosa et al. (1995), Tourinho (1995) e Tourinho (1996) tiveram como premissa fundamental o uso da equação de Cagan e técnicas econométricas importantes como a transformação Box-Cox e os testes de cointegração. Ainda, compreendendo-se o período no qual se inclui o Plano Real, destaca-se que poucas considerações foram identificadas, exceto aquelas concernentes à causalidade de Granger entre a demanda por moeda e a inflação, a exogeneidade da demanda por moeda e a discussão acerca da sua estabilidade. Os trabalhos de Arnosti (2003) e Canêdo-Pinheiro (2011) contribuíram com o uso de técnicas econométricas relevantes para o estudo do período de alta instabilidade como o Filtro de Kalman e testes de cointegração com quebras-estruturais endógenas.

Uma breve síntese permite inferir quatro pontos fundamentais sobre a literatura empírica sobre a demanda por moeda no Brasil. O primeiro deles é que, independente da frequência dos dados, o período compreendeu subperíodos de aceleração inflacionária nos quais se alternam expansão e contração do produto doméstico. Entretanto, o escopo para o estudo da estabilidade ou não da demanda por moeda no período pós-Plano Real é, ainda, uma lacuna importante para se corroborar os problemas identificados nos anos de aceleração inflacionária relativos ao início dos anos sessenta, fins dos anos setenta e o início dos anos oitenta até a execução do Plano Real.

O segundo ponto é que se credita o estudo da estabilidade da demanda por moeda e o estudo da adequação do modelo de Cagan ao período de inflação elevada, ou hiperinflação, os principais objetivos da análise do tema. Ainda, nota-se que, reiteradas vezes, ao se considerar o início dos anos oitenta, justifica-se a ampliação do conceito de moeda buscando-se a inclusão de ativos indexados na sua análise, seja ela desenvolvida teoricamente ou discutida tecnicamente com vistas às restrições impostas ao papel da política monetária.

A aplicação da transformação Box-Cox mostrou-se limitada à discussão do período de inflação elevada, mesmo uma vez conhecida a instabilidade da demanda por moeda. Suas extensões possibilitaram discussões quanto ao erro de especificação nos modelos, em detrimento do uso de modelos ad hoc, pouco presentes na literatura empírica brasileira. Considera-se este como o terceiro ponto.

Finalmente, o quarto ponto, é que do ponto de vista do instrumental econométrico, presente na literatura econométrica durante o período de estudos e publicações revisados, questiona-se o tardio e limitado uso de técnicas de séries temporais como os testes de raízes unitárias, testes de cointegração, e as especificações destes decorrentes.

Os resultados, em geral, apenas corroboraram o que a literatura teórica havia proposto, sem destes decorrerem contribuições significativas para novas considerações teóricas. Com base nesse ponto de vista, um escopo razoável tem sido a inclusão de novos agregados em uma função genérica e o estudo dos efeitos da substituição de ativos mais líquidos por ativos menos líquidos, principalmente tomando-se uma perspectiva intertemporal.

O que deve ser considerado é que a literatura empírica, excetuando-se o trabalho de Arnosti (2003), estudou apenas o período anterior ao Plano Real, 
cujo período não é analisado no presente trabalho.

\section{Modelo de Regressão Cointegrada}

A análise econométrica abordada que investiga a demanda por moeda está relacionada à aplicação de testes de estacionariedade e de cointegração. Quanto à forma funcional, existem evidências empíricas favoráveis à forma semi-log para a economia americana, conforme mostra Ireland (2009), e à forma log-log para dados brasileiros anteriores ao Plano Real, segundo Simonsen \& Cysne (2007), mesmo a literatura sugerindo que a forma log-log seria robusta para patamares inflacionários mais baixos, ver Serletis \& Yavari (2004). Entretanto, antes de mostrar e interpretar os resultados, a próxima subseção apresenta os dados utilizados nos exercícios empíricos.

\subsection{Os dados}

Os dados utilizados referem-se ao período pós-Plano Real, do quarto trimestre de 1994 ao quarto trimestre de 2011. Esse período coincide com implantação, pelo Instituto Brasileiro de Geografia e Estatística (IBGE), de significativas alterações metodológicas no Sistema de Contas Nacionais que dificultam a utilização dos dados obtidos diretamente no instituto. A série de contas nacionais do IBGE com nova referência ano 2000, chamada de "Sistema de Contas Nacionais Trimestrais (SCNT) Referência 2000", foi calculada para os dados a partir de 2000. "Para o período anterior a 2000 realizou-se a retropolação dos dados até 1995, estimando-se uma nova série de Tabelas de Recursos e Usos na classificação do sistema atualmente divulgado, mas encadeada com a nova série no ano 2000" (IBGE 2007). De acordo com o IBGE, a coerência metodológica entre a nova referência (2000) e a série anterior (referência 1985) permitiu essa retropolação da série de contas nacionais para o período de 1995 a 1999 a partir de um novo ano de referência que incorpora no cálculo dos agregados a nova estrutura das atividades econômicas e a nova estrutura de ponderação. Já, para o período de 1991 a 1994, apenas os dados trimestrais de PIB real (em índice) foram retropolados com a nova referência (2000) - ver IBGE (2007b).

Em função dessas questões levantadas, o indicador de renda nacional (PIB em valores constantes, acumulado em 12 meses, R \$ milhões de 2009) foi calculado da seguinte forma: utilizou-se o PIB a preços correntes de 2009 e, por meio do índice encadeado com a nova referência (2000), estimou-se a série a preços constantes para todo o período.

O indicador de deflator $(2009=100)$ usado neste trabalho foi estimado utilizando-se os dados do IBGE de variação real do PIB e de PIB nominal com a nova referência (2000) para o período de 1995 a 2009. Para 1994, como não há dados de PIB em valores correntes do SCNT referência 2000, utilizou-se os dados de variação real e valores correntes do PIB do SCNT referência de 1985 (série antiga) para se estimar o deflator.

$\mathrm{O}$ indicador de nível de moeda na economia escolhido foi o M1, média. O Banco Central do Brasil (BACEN) fornece esses dados com periodicidade mensal e em unidade de moeda corrente (u.m.c.). A série foi toda uniformizada, convertendo-se os dados de 1994 de cruzeiro real para real, e transformada em uma série trimestral por meio de médias aritméticas dos dados mensais. 
Por último, a taxa nominal de juros foi calculada por meio de uma média trimestral (anualizada) da série de dados da taxa Selic acumulada no mês, disponibilizada pelo BACEN.

\subsection{Resultados empíricos}

Para verificar estatisticamente se as formas funcionais log-log e semi-log, equações (1) e (2), se ajustam aos dados do Brasil pós-Plano Real, procedem-se, primeiramente, os testes de raiz unitária de Phillips-Perron com correção para problemas de autocorrelação serial, os testes Dickey-Fuller Generalized Least Squares (DFGLS), em que as séries são transformadas pelo método de mínimos quadrados generalizados, e os testes de raiz unitária com quebra estrutural de Zivot \& Andrews (1992); para então prosseguir investigando os modelos de regressão cointegrada ${ }^{3}$. A Tabela 1 apresenta os resultados dos testes de raiz unitária para as variáveis econômicas diretamente envolvidas no estudo: $\ln (m), \ln (r)$ e $r$, em que $m$ é igual a $M 1 / P I B, r$ a taxa de juros nominal e $\ln$ o logaritmo neperiano.

Tabela 1: Testes de raiz unitária Phillips-Perron com estimador NeweyWest

\begin{tabular}{|c|c|c|c|c|c|c|c|c|c|c|c|}
\hline \multicolumn{4}{|c|}{$\ln (m)$} & \multicolumn{4}{|c|}{$\ln (r)$} & \multicolumn{4}{|c|}{$r$} \\
\hline$\widehat{\alpha}$ & $\widehat{\rho}$ & $q$ & $z_{t}$ & $\widehat{\alpha}$ & $\widehat{\rho}$ & $q$ & $z_{t}$ & $\widehat{\alpha}$ & $\widehat{\rho}$ & $q$ & $z_{t}$ \\
\hline \multirow[t]{9}{*}{$-0,109$} & 0,961 & 0 & $-1,099$ & $-0,166$ & 0,916 & 0 & $-2,273$ & 0,021 & 0,867 & 0 & $-3,212$ \\
\hline & & 1 & $-1,270$ & & & 1 & $-2,315$ & & & 1 & $-3,185$ \\
\hline & & 2 & $-1,196$ & & & 2 & $-2,293$ & & & 2 & $-3,209$ \\
\hline & & 3 & $-1,113$ & & & 3 & $-2,273$ & & & 3 & $-3,268$ \\
\hline & & 4 & $-1,132$ & & & 4 & $-2,268$ & & & 4 & $-3,295$ \\
\hline & & 5 & $-1,169$ & & & 5 & $-2,267$ & & & 5 & $-3,297$ \\
\hline & & 6 & $-1,165$ & & & 6 & $-2,264$ & & & 6 & $-3,322$ \\
\hline & & 7 & $-1,142$ & & & 7 & $-2,264$ & & & 7 & $-3,380$ \\
\hline & & 8 & $-1,136$ & & & 8 & $-2,271$ & & & 8 & $-3,473$ \\
\hline
\end{tabular}

Fonte: Valores estimados pelos autores.

Obs.: Período amostral de 1994: 04 a $2011: 04$. Nos testes de raiz unitária

Phillips-Perron os valores críticos para o teste $z_{t}$ foram coletados em Hamilton (1994, p.

763, Tabela B.6), Caso 2): $-2,60(10 \%),-2,93(5 \%)$, e $-3,58(1 \%)$. Os testes

Phillips-Perron com correção de autocorrelação serial seguem os programas de Ireland (2009), disponibilizados no seu site: https: / / www2 . bc . edu/peter-ireland/ programs.html. As estimativas $\widehat{\alpha}$ e $\widehat{\rho}$ representam o intercepto e o coeficiente de inclinação da regressão de mínimos quadrados ordinários da variável em questão em relação a uma constante e seus próprios valores defasados; enquanto $z_{t}$ é a estatística Phillips-Perron corrigida para correlação serial, usando o estimador de Newey \& West (1987), e q é o valor de truncamento da defasagem.

Analisando, inicialmente, o teste de Phillips-Perron, ao comparar os valores calculados com os valores críticos, observa-se a não rejeição da hipótese nula, em nenhum dos casos, ao nível de 1\% de significância, ou seja, as séries não são estacionárias, são $I(1)$. Esse resultado permite seguir para a investigação de cointegração; entretanto, ao nível de 5\% de significância, a taxa nominal de juros seria estacionária, $I(0)$. Logo, a este último nível de significância somente seria viável seguir analisando a forma funcional log-log. Para gerar

\footnotetext{
${ }^{3}$ Pressupõe-se que as equações lineares relacionam variáveis $\ln (m)$ e $\ln (r)$, em um caso, e $\ln (m)$ e $r$, no outro; ver Anderson \& Rasche (2001).
} 
maior robustez aos resultados, a Tabela 2 apresenta mais duas análises, uma por meio de testes DFGLS no qual corroboram o fato das séries serem I(1), e outra usando testes de quebra estrutural de Zivot e Andrews, cujos resultados mostram apenas a taxa de juros em nível como $I(0)$, mesmo ao nível de $1 \%$ de significância.

Tabela 2: Testes de raiz unitária DFGLS e Testes de raiz unitária Zivot \& Andrews

\begin{tabular}{|c|c|c|c|c|c|c|c|c|c|c|c|}
\hline \multicolumn{6}{|c|}{ DFGLS } & \multicolumn{6}{|c|}{ Zivot \& Andrews } \\
\hline & $(m)$ & & $n(r)$ & & $r$ & & $(m)$ & & $l(r)$ & & $r$ \\
\hline \multicolumn{6}{|c|}{ Com intercepto e tendência } & \multicolumn{6}{|c|}{ Quebra no intercepto e tendência } \\
\hline Lag & t-Stat. & Lag & t- Stat. & Lag & t- Stat. & Lag & t- Stat. & Lag & t-Stat. & Lag & t- Stat. \\
\hline 09 & $-1,546$ & 02 & $-2,488$ & 08 & $-1,094$ & 09 & $-2,252$ & 03 & $-4,533$ & 04 & $-5,751$ \\
\hline \multicolumn{6}{|c|}{ Com intercepto } & \multicolumn{6}{|c|}{ Quebra no intercepto } \\
\hline Lag & t-Stat. & Lag & t- Stat. & Lag & t- Stat. & Lag & t- Stat. & Lag & t-Stat. & Lag & t- Stat. \\
\hline 09 & $-0,482$ & 02 & $-0,236$ & 02 & $-0,541$ & 09 & $-2,324$ & 03 & $-4,726$ & 04 & $-6,108$ \\
\hline
\end{tabular}

Fonte: Valores calculados pelos autores.

Obs.: Nos testes de raiz unitária DFGLS foram usados o critério de seleção AIC modificado (MAIC), usando o software EViews 7, com níveis de significância $-2,86$ $(10 \%),-3.16(5 \%)$, e $-3.74(1 \%)$ com intercepto e tendência na equação de teste, e com níveis de significância -1,61 (10\%), -1,95 (5\%), e -2,60 (1\%) somente com intercepto. Nos testes de raiz unitária com quebra estrutural no intercepto, os níveis de significância foram de $-4,58(10 \%),-4,93(5 \%)$ e $-5,34(1 \%)$. Ainda, os testes indicaram quebras em 1999:03 para a série $\operatorname{Ln}(\mathrm{m})$, em 2002:04 para Ln(r) e 1999:03 para a série r. Já nos testes com quebra no intercepto e tendência os níveis críticos foram $-4,82(10 \%),-5,08(5 \%)$ e $-5,57(1 \%)$, com quebras em 2007:03 para a série $\operatorname{Ln}(\mathrm{m})$, em 2002:03 para $\operatorname{Ln}(\mathrm{r})$ e 2002:04 para a série r.

Adota-se, no próximo passo, o teste de cointegração Phillips-Ouliaris. A Tabela 3 mostra os valores calculados para $z_{t}$ que comparados aos valores tabelados obtidos, ao nível de significância de 5\%, revelam a rejeição da hipótese nula, em ambos os casos, ou seja, as séries são cointegradas nas duas formas funcionais; cabe lembrar que o nível da taxa nominal de juros poderia até ser não estacionário, se desconsiderar o teste com quebra estrutural aplicado e levar em consideração nível de significância de $1 \%$.

Mas, a este último nível de significância os resultados são inconclusivos no teste de cointegração, uma vez que a rejeição da hipótese nula, em ambos os casos, ocorreria se considerar um valor q maior que seis $(q>6)$.

O resultado acima pode ter sido gerado na presença de quebras estruturais cujas mudanças podem ter afetado a estabilidade dos parâmetros do vetor de cointegração. Diante disso, propõe-se o teste de Gregory \& Hansen (1996), que permite investigar a cointegração em modelos com mudanças de regime por meio de testes baseados nos resíduos, ou seja, testa-se a hipótese nula de ausência de cointegração contra a hipótese alternativa de cointegração com ou sem mudança de regime em determinado período desconhecido. A Tabela 4 mostra os resultados dos testes de cointegração de Gregory e Hansen para as duas formas funcionais, onde se observa evidências da existência de cointegração entre as séries econômicas.

Como ambas as especificações estimadas impõem uma restrição de elasticidade-renda da moeda igual à unidade, testa-se novamente as mesmas especificações flexibilizando-se a hipótese. Nesse caso, aplica-se o teste Phillips- 
Tabela 3: Teste de cointegração Phillips-Ouliaris

\begin{tabular}{|c|c|c|c|c|c|c|c|c|c|}
\hline \multicolumn{10}{|c|}{ Amostra: 1994:04-2011:04 } \\
\hline \multicolumn{5}{|c|}{$\ln (m)=\alpha-\beta \ln (r)$} & \multicolumn{5}{|c|}{$\ln (m)=\alpha-\beta r$} \\
\hline$\hat{\alpha}$ & $\widehat{\beta}$ & $\hat{\rho}$ & Q & $\mathrm{z}_{t}$ & $\hat{\alpha}$ & $\widehat{\beta}$ & $\hat{\rho}$ & $\mathrm{q}$ & $\mathrm{z}_{t}$ \\
\hline$-3,6551$ & 0,4227 & 0,7535 & 0 & $-3,4858$ & -2.6098 & 1,5929 & 0,7643 & 0 & $-3,6073$ \\
\hline & & & 1 & $-3,7015$ & & & & 1 & $-3,7482$ \\
\hline & & & 2 & $-3,6877$ & & & & 2 & $-3,7525$ \\
\hline & & & 3 & $-3,7266$ & & & & 3 & $-3,8059$ \\
\hline & & & 4 & $-3,8485$ & & & & 4 & $-3,9241$ \\
\hline & & & 5 & $-3,9425$ & & & & 5 & $-4,0169$ \\
\hline & & & 6 & $-3,9703$ & & & & 6 & $-4,0506$ \\
\hline & & & 7 & $-3,9960$ & & & & 7 & $-4,0871$ \\
\hline & & & 8 & $-4,0405$ & & & & 8 & $-4,1347$ \\
\hline
\end{tabular}

Fonte: Valores estimados pelos autores.

Obs.: Utilizam-se mínimos quadrados ordinários para estimar o intercepto e o coeficiente de inclinação das relações funcionais (1) e (2), para, então, aplicar o teste de raiz unitária de Phillips \& Perron (1998), corrigido para correlação serial; verificando, assim, se os resíduos da regressão dessas equações são estacionários ou não. Em caso de rejeição da hipótese nula de presença de raiz unitária, em ambos os casos, tais resultados indicariam que (1) e (2) representam relações de cointegração, ou seja, existe uma combinação linear estacionária entre as duas variáveis não estacionárias em questão.

Ouliaris na forma funcional que relaciona o logaritmo neperiano dos saldos monetários reais, $\ln (M / P)$, ao logaritmo neperiano da renda real, $\ln (Y / P)$, e ao logaritmo neperiano da taxa nominal de juros, $\ln (r)$, usando o deflator implícito do PIB, $P$, para converter as séries nominais em reais. Posteriormente, modifica-se, em outro caso, apenas a taxa de juros que é considerada em nível, conforme destacado na Tabela 5. Nessa última tabela, observa-se a não rejeição da hipótese nula de ausência de cointegração entre a demanda por moeda e a taxa de juros, em ambos os casos, aos níveis de significância de $1 \%$ e 5\%. Tal resultado também acontece com Ireland (2009).

Novamente, cabe destacar, que este resultado pode ter sido afetado por quebras estruturais. Portanto, apresenta-se, na Tabela 6, os resultados de testes de cointegração de Gregory e Hansen para as duas formas funcionais, onde pode-se observar evidências da existência de cointegração entre as séries econômicas aos níveis de significância de 1 e $5 \%$.

Entretanto, os resultados acima teriam que pressupor séries $I(1)$, fato que não é robusto para a série da taxa de juros em nível quando se considera a quebra estrutural que foi relevante nos testes de cointegração. Portanto, segue-se considerando a forma log-log, sendo a forma funcional adequada aos dados brasileiros. Esse resultado revela para o período posterior à introdução do Plano Real evidência empírica de uma relação de demanda por moeda com elasticidade-juros constante de 0,423 (em valor absoluto), enquanto Simonsen \& Cysne (2007) apresentam elasticidade-juros de 0,525. Tal resultado, a princípio, destaca pequena diferença entre os dois ambientes. O inflacionário, analisado por Simonsen \& Cysne (2007), período de 1947-1993, enquanto o presente trabalho analisa um período de baixa inflação, 1995-2011. Em outras palavras, ambos mostram menor sensibilidade da demanda por moeda em relação aos juros.

Apesar da ausência de cointegração, a Tabela 5 chama atenção para a estimativa $\hat{\beta}_{y} \cong 1.58$ para o parâmetro da elasticidade-renda da moeda, o que excede a unidade, e uma estimativa $\hat{\beta}_{r} \cong 0,31$ para o parâmetro da elasticidadejuros, ou seja, inferior ao modelo cuja elasticidade-renda da moeda unitária é 
Tabela 4: Teste de cointegração com mudança de regimes

\begin{tabular}{|c|c|c|c|}
\hline \multicolumn{4}{|c|}{ Amostra: 1994:04 - 2011:04 } \\
\hline \multirow{2}{*}{\multicolumn{2}{|c|}{$\begin{array}{l}\ln (m)=\alpha-\beta \ln (r) \\
\text { t-statistic (level shift) }\end{array}$}} & \multirow{2}{*}{\multicolumn{2}{|c|}{$\begin{array}{c}\ln (m)=\alpha-\beta r \\
\text { t-statistic (level shift) }\end{array}$}} \\
\hline & & & \\
\hline$Z_{\alpha}^{*}$ & $-35,397$ & $Z_{\alpha}^{*}$ & $-29,500$ \\
\hline$Z_{t}^{*}$ & $-5,658^{* * *}$ & $Z_{t}^{*}$ & $-4,892^{* *}$ \\
\hline$A D F^{*}$ & $-6,035^{* * *}(8$ lags $)$ & $A D F$ & $-5,027^{* *}$ (8 lags) \\
\hline \multicolumn{2}{|c|}{ t-statistic (level shift with trend) } & \multicolumn{2}{|c|}{ t-statistic (level shift with trend) } \\
\hline$Z_{\alpha}^{*}$ & $-43,662^{*}$ & $Z_{\alpha}^{*}$ & $-43,952^{*}$ \\
\hline$Z_{t}^{*}$ & $-6,430^{* * *}$ & $Z_{t}^{*}$ & $-6,361^{* * *}$ \\
\hline$A D F$ & $-4,765^{*}$ (5 lags) & $A D F$ & $-4,863^{*}$ (5 lags) \\
\hline \multicolumn{2}{|c|}{ t-statistic (regime shift) } & \multicolumn{2}{|c|}{ t-statistic (regime shift) } \\
\hline$Z_{\alpha}^{*}$ & $-42,510^{*}$ & $Z_{\alpha}^{*}$ & $-45,569^{*}$ \\
\hline$Z_{t}^{*}$ & $-5,961^{* * *}$ & $Z_{t}^{*}$ & $-6,189^{* * *}$ \\
\hline$A D F$ & $-6,036^{* * *}(6$ lags $)$ & $A D F$ & $-5,731^{* * *}(8$ lags $)$ \\
\hline \multicolumn{4}{|c|}{ 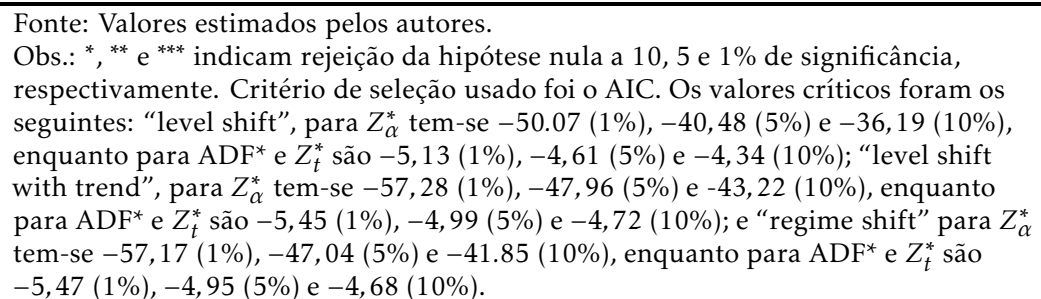 } \\
\hline
\end{tabular}

imposta. Diante desses resultados, e em busca de maior robustez, considera-se agora apenas a especificação log-log na aplicação do "dynamic OLS” (DOLS), testando a hipótese de elasticidade-renda unitária. A hipótese nula pressupõe que a mudança relativa na demanda por moeda é equivalente à mudança relativa na renda real. Cabe destacar que as regressões dinâmicas assumem que as variáveis não estacionárias $\ln (m)$ e $\ln (r)$ são cointegradas. Ao contrário das regressões estáticas da Tabela 3, estas não podem ser usadas para investigar a hipótese de ausência ou não de cointegração ${ }^{4}$. A Tabela 7 reporta as estimativas DOLS para o intercepto, $\hat{\alpha}$, e o coeficiente de inclinação, $\hat{\beta}$, das relações de cointegração, os erros padrão, se $(\hat{\beta})$, computados usando o estimador de Newey e West para diferentes p e q. Nota-se certa semelhança no modelo log-log comparado aos resultados da Tabela 3, mas com o valor absoluto da elasticidade-juros maior e estatisticamente significativo, conforme estatística t, $\hat{\beta} / s e(\hat{\beta})$, para a elasticidade-juros da moeda. Entretanto, esses resultados se aproximam muito mais ao apontado por Simonsen \& Cysne (2007).

Relaxando-se o pressuposto de elasticidade-renda da moeda unitária, a Tabela 7 reporta as respectivas estimativas $\hat{\alpha}, \hat{\beta}_{y}$ e $\hat{\beta}_{r}$ da especificação que relaciona a variável resposta $\ln (M / P)$ às variáveis explicativas $\ln (Y / P)$ e $\ln (r)$. Ainda, a tabela mostra os erros-padrão, $\operatorname{se}\left(\hat{\beta}_{y}\right)$ e $\operatorname{se}\left(\hat{\beta}_{r}\right)$, corrigidos para autocorrelação usando estimador de Newey e West, e a correspondente estatística Wald que permite testar a hipótese nula de elasticidade-renda de longo prazo unitária, $\mathrm{W}\left(\beta_{y}=1\right)$. Comparando as estatísticas calculadas com as tabeladas, observa-se que a hipótese nula de elasticidade-renda unitária, dentro da especificação log-log, não é rejeitada ao nível de significância de 5\%. Logo, pode-se

\footnotetext{
${ }^{4}$ Ver Stock \& Watson (1993) e Hamilton (1994).
} 
Tabela 5: Teste de cointegração Phillips-Ouliaris (1990)

\begin{tabular}{|c|c|c|c|c|c|c|c|c|c|c|c|}
\hline \multicolumn{12}{|c|}{ Amostra: 1994:04-2011:04 } \\
\hline \multicolumn{6}{|c|}{$\ln (M / P)=\alpha+\beta_{y} \ln (Y / P)-\beta_{r} \ln (r)$} & \multicolumn{6}{|c|}{$\ln (M / P)=\alpha+\beta_{y} \ln (Y / P)-\beta_{r} r$} \\
\hline$\widehat{\alpha}$ & $\widehat{\beta}_{v}$ & $\widehat{\beta_{r}}$ & $\overline{\widehat{\rho}}$ & $\mathrm{Q}$ & $\mathrm{z}_{t}$ & $\widehat{\alpha}$ & $\widehat{\beta_{v}}$ & $\widehat{\beta_{r}}$ & $\widehat{\bar{\rho}}$ & $q$ & $\mathrm{z}_{t}$ \\
\hline$-12,1333$ & 1,5827 & 0,3137 & 0,8202 & 0 & $-2,5483$ & $-14,3556$ & 1,7843 & 1,1075 & 0,7795 & 0 & $-2,5483$ \\
\hline & & & & 1 & $-2,6159$ & & & & & 1 & $-2,6159$ \\
\hline & & & & 2 & $-2,6359$ & & & & & 2 & $-2,6359$ \\
\hline & & & & 3 & $-2,6266$ & & & & & 3 & $-2,6266$ \\
\hline & & & & 4 & $-2,7513$ & & & & & 4 & $-2,7513$ \\
\hline & & & & 5 & $-2,8135$ & & & & & 5 & $-2,8135$ \\
\hline & & & & 6 & $-2,8782$ & & & & & 6 & $-2,8782$ \\
\hline & & & & 7 & $-2,9130$ & & & & & 7 & $-2,9130$ \\
\hline & & & & 8 & $-2,9561$ & & & & & 8 & $-2,9561$ \\
\hline
\end{tabular}

Obs.: Valores críticos para $z_{t}$ foram coletados em Hamilton (1994, p. 766, Tabela B.9, Caso 3), indicando: $-3,52(10 \%)$; $-3,80$ (5\%), e $-4,36(1 \%)$ 
Tabela 6: Teste de cointegração com mudança de regimes

\begin{tabular}{|c|c|c|c|}
\hline \multicolumn{4}{|c|}{ Amostra: 1994:04-2011:04 } \\
\hline $\ln (M)$ & $\chi+\beta_{y} \ln (Y / P)-\beta_{r} \ln (r)$ & $\ln (N$ & $=\alpha+\beta_{y} \ln (Y / P)-\beta_{r} r$ \\
\hline \multicolumn{2}{|c|}{ t-statistic (level shift) } & \multicolumn{2}{|c|}{ t-statistic (level shift) } \\
\hline$Z_{\alpha}^{*}$ & $-44,823^{* *}$ & $Z_{\alpha}^{*}$ & $-44,242^{* *}$ \\
\hline$Z_{t}^{*}$ & $-5,910^{* * *}$ & $Z_{t}^{*}$ & $-5,937^{* * *}$ \\
\hline$A D F^{*}$ & $-5,708^{* * *}(8$ lags $)$ & $A D F$ & $-5,844^{* * *}$ (8 lags) \\
\hline \multicolumn{2}{|c|}{ t-statistic (level shift with trend) } & \multicolumn{2}{|c|}{ t-statistic (level shift with trend) } \\
\hline$Z_{\alpha}^{*}$ & $-52,809^{* *}$ & $Z_{\alpha}^{*}$ & $-51,446^{* *}$ \\
\hline$Z_{t}^{*}$ & $-7,071^{* * *}$ & $Z_{t}^{*}$ & $-6,910^{* * *}$ \\
\hline$A D F$ & $-5,816^{* * *}(1 \mathrm{lag})$ & $A D F$ & $-5,631^{* * *}(1 \mathrm{lag})$ \\
\hline \multicolumn{2}{|c|}{ t-statistic (regime shift) } & \multicolumn{2}{|c|}{ t-statistic (regime shift) } \\
\hline$Z_{\alpha}^{*}$ & $-52,593^{* *}$ & $Z_{\alpha}^{*}$ & $-52,445^{* *}$ \\
\hline$Z_{t}^{*}$ & $-6,850^{* * *}$ & $Z_{t}^{*}$ & $-6,842^{* * *}$ \\
\hline$A D F$ & $-5,485^{* * *}(0 \mathrm{lag})$ & $A D F$ & $-5,770^{* * *}(0 \mathrm{lag})$ \\
\hline
\end{tabular}

Obs.: ${ }^{*},{ }^{* *} \mathrm{e}^{* * *}$ indicam rejeição da hipótese nula a 10,5 e 1\% de significância, respectivamente. Critério de seleção usado foi o AIC. Os valores críticos foram os seguintes: "level shift", para $Z_{\alpha}^{*}$ tem-se $-50,07(1 \%),-40,48(5 \%)$ e $-36,19(10 \%)$, enquanto para $\mathrm{ADF}^{*}$ e $Z_{t}^{*}$ são $-5,13(1 \%),-4,61(5 \%)$ e $-4,34$ $(10 \%)$; "level shift with trend", para $Z_{\alpha}^{*}$ tem-se $-57,28(1 \%),-47,96(5 \%)$ e $-43,22$ (10\%), enquanto para $\mathrm{ADF}^{*}$ e $Z_{t}^{*}$ são -5, $45(1 \%),-4,99(5 \%)$ e $-4,72$ $(10 \%)$; e "regime shift" para $Z_{\alpha}^{*}$ tem-se $-57,17(1 \%),-47,04(5 \%)$ e $-41,85$

(10\%), enquanto para $\mathrm{ADF}^{*}$ e $Z_{t}^{*}$ são $-5,47(1 \%),-4,95(5 \%)$ e $-4,68(10 \%)$.

Tabela 7: OLS Dinâmico

\begin{tabular}{|c|c|c|c|c|c|c|c|c|c|c|c|c|}
\hline \multicolumn{13}{|c|}{ Amostra: 1994:04-2011:04 } \\
\hline \multicolumn{5}{|c|}{$\ln (m)=\alpha-\beta \ln (r)$} & \multicolumn{8}{|c|}{$\ln (M / P)=\alpha+\beta_{y} \ln (Y / P)-\beta_{r} \ln (r)$} \\
\hline$\widehat{\alpha}$ & $\widehat{\beta}$ & s.e. $(\widehat{\beta})$ & p & q & $\widehat{\alpha}$ & $\widehat{\beta}_{y}$ & s.e. $\left(\widehat{\beta}_{y}\right)$ & $\widehat{\beta}_{r}$ & s.e. $\left(\widehat{\beta}_{r}\right)$ & $\mathrm{p}$ & q & $W\left(\beta_{\nu}\right)=1$ \\
\hline \multirow[t]{4}{*}{$-3,7724$} & 0,4955 & 0,0526 & 1 & 2 & $-8,5128$ & 1,3294 & 0,3492 & 0,4276 & 0,1310 & . & 2 & 0,8896 \\
\hline & & 0,0632 & & 4 & & & 0,4242 & & 0,1591 & & 4 & 0,6029 \\
\hline & & 0,0700 & & 6 & & & 0,4765 & & 0,1787 & & 6 & 0,4778 \\
\hline & & 0,0740 & & 8 & & & 0,5113 & & 0,1918 & & 8 & 0,4150 \\
\hline \multirow[t]{4}{*}{$-3,7784$} & 0,5042 & 0,0527 & 2 & 2 & $-7,2823$ & 1,2460 & 0,3854 & 0,4138 & 0,1448 & 2 & 2 & 0,4073 \\
\hline & & 0,0629 & & 4 & & & 0,4588 & & 0,1723 & & 4 & 0,2875 \\
\hline & & 0,0700 & & 6 & & & 0,5122 & & 0,1924 & & 6 & 0,2307 \\
\hline & & 0,0744 & & 8 & & & 0,5464 & & 0,2053 & & 8 & 0,2027 \\
\hline \multirow[t]{4}{*}{$-3,7829$} & 0,5123 & 0,0496 & 3 & 2 & 0,8332 & 0,6753 & 0,3879 & 0,5642 & 0,1385 & 3 & 2 & 0,7007 \\
\hline & & 0,0582 & & 4 & & & 0,4288 & & 0,1531 & & 4 & 0,5734 \\
\hline & & 0,0643 & & 6 & & & 0,4609 & & 0,1646 & & 6 & 0,4965 \\
\hline & & 0,0689 & & 8 & & & 0,4856 & & 0,1734 & & 8 & 0,4471 \\
\hline
\end{tabular}

Fonte: Valores estimados pelos autores.

Obs.: Valores críticos para $W\left(\beta_{y}=1\right)$ foram coletados em Hamilton (1994, Tabela B.2, p.754), indicando: $2,71(10 \%) ; 3,84(5 \%)$, e 6,63 (1\%), 
dizer que as mudanças relativas na demanda por moeda seriam inferiores às mudanças relativas na taxa de juros.

Em síntese, os resultados acima corroboram a forma funcional log-log restrita para os dados brasileiros pós-Plano Real utilizados na estimação da demanda por moeda. Baseado nessa especificação apresenta-se, na próxima seção, as estimativas dos custos sociais da inflação no Brasil gerados a partir das correspondentes fórmulas funcionais (3) e (5).

\section{Estimativas dos Custos de Bem-Estar da Inflação}

Nesta seção, estimam-se os custos de bem-estar da inflação no Brasil pósplano Real a partir das aproximações de Bailey (1956), equação (3), e Simonsen \& Cysne (2007), equação (5), que seguem apresentados nas Tabelas 8 e 9, respectivamente.

Seguindo Ireland (2009), pode-se considerar, a princípio, o custo de bemestar de uma inflação de $10 \%$, o que equivale a analisar a diferença $W(0,13)$ - W(0,03). Com esse nível de inflação, a forma funcional log-log indica na Tabela 8 um custo social da inflação entre 0,33 e $0,45 \%$ da renda nacional. Ao se considerar uma inflação de $2 \%$, os custos reduzem para algo entre 0,09 e $0,12 \%$ da renda, e assim poderia continuar propondo diferentes níveis inflacionários. Apesar do mercado recentemente estar trabalhando com níveis menores de taxa de juros real neutra no Brasil, dificilmente a taxa real de juros de estado estacionário no país estaria no mesmo patamar de países desenvolvidos neste período em estudo, ou seja, $3 \%$ ao ano. Conforme Caetano et al. (2012), quando questionadas pelo Banco Central do Brasil (BCB) sobre qual valor sua instituição considerava como sendo a atual taxa de juros real de equilíbrio/neutra no Brasil, as instituições responderam em novembro de 2010 valores cuja mediana ficou em $6,75 \%$ a.a. (em média, $6,55 \%$ a.a.), enquanto a estimativa destes autores foi de 5,61\% ao ano (em média, 5,64\% a.a.). Comparada com a pesquisa de fevereiro de 2012 do BCB, observa-se respostas menores dadas pelo mercado, mediana de 5,43\% a.a. (em média, $5,50 \%$ a.a.), enquanto a estimativa dos autores para dezembro de 2011, fim da amostra deles, ficou em 5,76\% a.a. (em média, 5,78\% a.a.).

Assim sendo, seria razoável também considerar alternativas para o nível da taxa de juros real anual de estado estacionário para a economia brasileira, tais como: 5, 7 e 9\%, para, em seguida, analisar o comportamento do custo de bem-estar frente a uma inflação de 2, 4,5, 7 e 10\% ao ano, respectivamente, sendo que a atual meta de inflação estipulada pelo Conselho Monetário Nacional está em 4,5\% ao ano. A Tabela 8 , Painéis B a D mostram custos de bem-estar da inflação decrescentes quando se considera taxa real de juros de estado estacionário crescente. Tal resultado ocorre devido à diferença aparecer na taxa de inflação, ou seja, trata-se um ganho de nível menor de inflação quando se faz este aumento na taxa neutra. Entretanto, tal resultado decrescente dos custos sociais não pode ser confundido com os efeitos negativos de se manter uma taxa real de juros de estado estacionário maior. Ou seja, não basta reduzir a taxa nominal de juros dentro da economia, mas também reduzir a taxa real de juros neutra condizente com um crescimento sustentado.

Para uma inflação média anual de aproximadamente $4,5 \%$ ao ano, conforme a atual meta de inflação, o custo de bem-estar estaria entre 0,1506 e $0,2038 \%$ da renda, considerando uma taxa real de juros anual de estado es- 
Tabela 8: Custos de Bem-Estar da Inflação com aproximação de Bailey

\begin{tabular}{|c|c|c|c|c|c|}
\hline \multicolumn{6}{|c|}{ Painel A } \\
\hline \multicolumn{6}{|c|}{ Custo Social (em \% da renda) } \\
\hline & & & Inflação 0\% & Inflação 2\% & Inflação $10 \%$ \\
\hline Regressão & $A=\exp (\widehat{\alpha})$ & $\eta=\widehat{\beta}$ & $\mathrm{W}(0,03)$ & $\mathrm{W}(0,05)$ & $\mathrm{W}(0,13)$ \\
\hline OLS estático (Tabela 2) & 0,0259 & 0,4227 & 0,2501 & 0,3359 & 0,5831 \\
\hline OLS dinâmico, $\mathrm{p}=1$ (Tabela 4 ) & 0,0230 & 0,4955 & 0,3851 & 0,4983 & 0,8069 \\
\hline OLS dinâmico, $\mathrm{p}=2$ (Tabela 4$)$ & 0,0229 & 0,5042 & 0,4086 & 0,5264 & 0,8454 \\
\hline OLS dinâmico, $\mathrm{p}=3$ (Tabela 4$)$ & 0,0228 & 0,5123 & 0,4323 & 0,5546 & 0,8838 \\
\hline \multicolumn{6}{|c|}{ Painel B } \\
\hline & Inflação $0 \%$ & Inflação 2\% & Inflação 4.5\% & Inflação 7\% & Inflação $10 \%$ \\
\hline Regressão & $\mathrm{W}(0,05)$ & $\mathrm{W}(0,07)$ & $\mathrm{W}(0,095)$ & $\mathrm{W}(0,12)$ & $\mathrm{W}(0,15)$ \\
\hline OLS estático (Tabela 2) & 0,3359 & 0,4079 & 0,4865 & 0,5567 & 0,6333 \\
\hline OLS dinâmico, $\mathrm{p}=1$ (Tabela 4 ) & 0,4983 & 0,5905 & 0,6888 & 0,7750 & 0,8673 \\
\hline OLS dinâmico, $\mathrm{p}=2$ (Tabela 4$)$ & 0,5264 & 0,6220 & 0,7236 & 0,8125 & 0,9075 \\
\hline OLS dinâmico, $\mathrm{p}=3$ (Tabela 4 ) & 0,5546 & 0,6535 & 0,7584 & 0,8500 & 0,9477 \\
\hline \multicolumn{6}{|c|}{ Painel C } \\
\hline & Inflação $0 \%$ & Inflação 2\% & Inflação $4.5 \%$ & Inflação 7\% & Inflação $10 \%$ \\
\hline Regressão & $\mathrm{W}(0,07)$ & $\mathrm{W}(0,09)$ & $\mathrm{W}(0,115)$ & $\mathrm{W}(0,14)$ & $\mathrm{W}(0,17)$ \\
\hline OLS estático (Tabela 2) & 0,4079 & 0,4715 & 0,5432 & 0,6086 & 0,6807 \\
\hline OLS dinâmico, $\mathrm{p}=1$ (Tabela 4$)$ & 0,5905 & 0,6703 & 0,7585 & 0,8377 & 0,9239 \\
\hline OLS dinâmico, $\mathrm{p}=2($ Tabela 4$)$ & 0,6220 & 0,7045 & 0,7955 & 0,8770 & 0,9656 \\
\hline OLS dinâmico, $\mathrm{p}=3$ (Tabela 4 ) & 0,6535 & 0,7387 & 0,8325 & 0,9163 & 1,0073 \\
\hline \multicolumn{6}{|c|}{ Painel D } \\
\hline & Inflação $0 \%$ & Inflação 2\% & Inflação $4,5 \%$ & Inflação 7\% & Inflação 10\% \\
\hline Regressão & $\mathrm{W}(0,09)$ & $\mathrm{W}(0,11)$ & $\mathrm{W}(0,135)$ & $\mathrm{W}(0,16)$ & $\mathrm{W}(0,19)$ \\
\hline OLS estático (Tabela 2) & 0,4715 & 0,5295 & 0,5959 & 0,6573 & 0,7259 \\
\hline OLS dinâmico, $\mathrm{p}=1$ (Tabela 4$)$ & 0,6703 & 0,7417 & 0,8224 & 0,8960 & 0,9772 \\
\hline OLS dinâmico, $\mathrm{p}=2($ Tabela 4$)$ & 0,7045 & 0,7782 & 0,8613 & 0,9370 & 1,0204 \\
\hline OLS dinâmico, $\mathrm{p}=3$ (Tabela 4 ) & 0,7387 & 0,8146 & 0,9002 & 0,9780 & 1,0635 \\
\hline
\end{tabular}

Fonte: Valores estimados pelos autores.

Obs.: Os custos de bem-estar da inflação calculados assumem taxas real de juros de estado estacionário

equivalentes à: i) Painel $A, r=0,03$; ii) Painel $B, r=0,05$; iii) Painel $C, r=0,07$; e Painel $D, r=0,09$, sob

pressuposto de inflação nula ou estabilidade de preços. 
tacionário de $5 \%$. Se a taxa real neutra é de $7 \%$ a.a., os custos seriam algo entre 0,1354 e $0,1790 \%$ da renda. Esse exemplo mostra claramente a conclusão acima, isto é, o ganho em termos de bem-estar ao reduzir a inflação será tanto maior quanto menor for a taxa real neutra de juros doméstica.

A Tabela 9 apresenta as estimativas dos custos de bem-estar da inflação usando a aproximação de Simonsen e Cysne, onde a mesma situa-se mais próxima da solução da equação original do que a aproximação dada pela fórmula de Bailey. Usando a demanda por moeda log-log, equação (1), estimada, podese obter os custos sociais a partir da equação (5).

Na Tabela 9, os Painéis B a D mostram os resultados onde também se observa os mesmos comportamentos dos custos de bem-estar da inflação quando se considera taxa real de juros de estado estacionário crescente.

Analisando novamente o caso de uma inflação média de aproximadamente $4,5 \%$ ao ano, o custo de bem-estar estaria entre 0,1498 e $0,2026 \%$ da renda, considerando uma taxa real de juros anual de estado estacionário de $5 \%$. Se a taxa real neutra é de 7\% a.a., os custos seriam algo entre 0,1345 e 0,1778\% da renda. Nota-se que o mesmo raciocínio anterior é valido aqui.

Conforme Simonsen \& Cysne (2007), as estimativas de custos de bem-estar baseadas nas hipóteses de Bailey sempre levam a uma superestimativa dos custos sociais da inflação. Logo, é esperado os resultados serem inferiores ao da aproximação de Bailey, apesar de a diferença não ser discrepante.

As Figuras 1 e 2 apresentam os custos sociais para uma taxa real neutra de juros de $7 \%$ ao ano dado certo nível de taxa média de inflação ao ano tanto para a aproximação de Bailey quanto de Simonsen e Cysne. A conclusão após visualização gráfica não surpreende, ou seja, quanto maior a inflação maior o custo social.

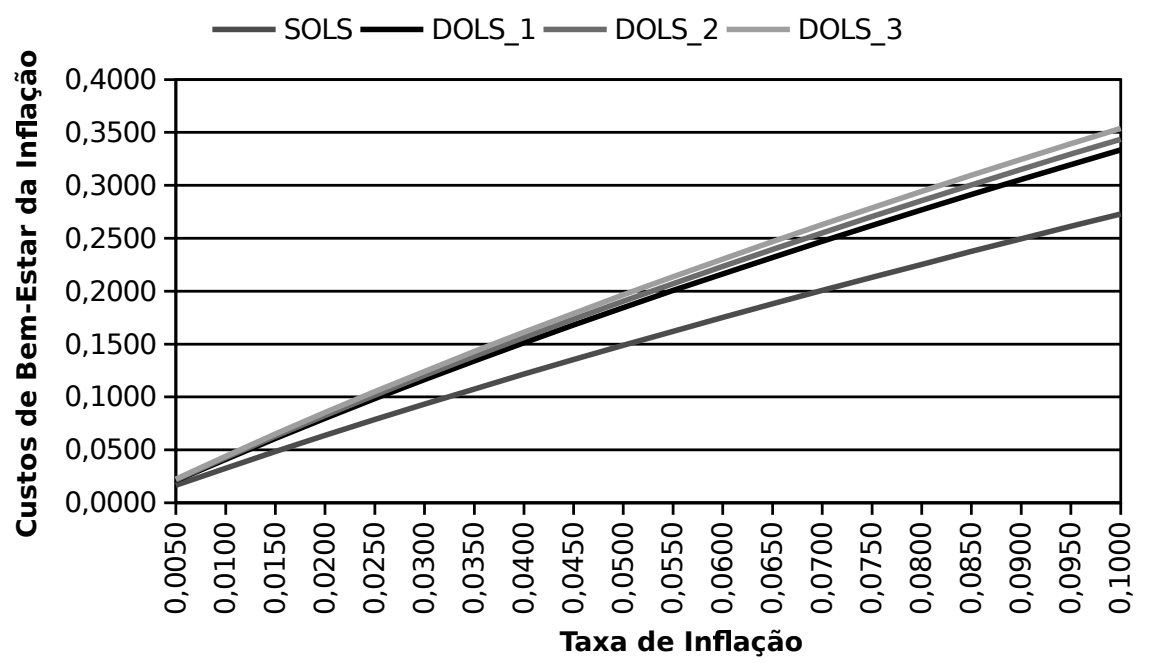

Fonte: Elaboração própria.

Figura 1: Custos de Bem-Estar da Inflação com aproximação de Bailey

Conforme a teoria, tal custo social só será zerado se a taxa nominal de juros também for anulada. Logo, conforme Friedman, a otimização do padrão monetário não é condicionada à estabilidade dos preços, mas sim a um processo de deflacionamento que conduz à equivalência da taxa real de juros com 
Tabela 9: Custos de Bem-Estar da Inflação com aproximação de Simonsen e Cysne

\begin{tabular}{|c|c|c|c|c|c|}
\hline \multicolumn{6}{|c|}{ Painel A } \\
\hline \multicolumn{6}{|c|}{ Custo Social (em \% da renda) } \\
\hline & & & Inflação0\% & Inflação2\% & Inflação10\% \\
\hline Regressão & $A=\exp (\widehat{\alpha})$ & $\eta=\widehat{\beta}$ & $\mathrm{W}(0,03)$ & $\mathrm{W}(0,05)$ & $\mathrm{W}(0,13)$ \\
\hline OLS estático (Tabela 2) & 0,0259 & 0,4227 & 0,2497 & 0,3351 & 0,5808 \\
\hline OLS dinâmico, $\mathrm{p}=1$ (Tabela 4) & 0,0230 & 0,4955 & 0,3843 & 0,4970 & 0,8036 \\
\hline OLS dinâmico, $\mathrm{p}=2$ (Tabela 4 ) & 0,0229 & 0,5042 & 0,4078 & 0,5250 & 0,8419 \\
\hline OLS dinâmico, $\mathrm{p}=3$ (Tabela 4 ) & 0,0228 & 0,5123 & 0,4314 & 0,5531 & 0,8801 \\
\hline \multicolumn{6}{|c|}{ Painel B } \\
\hline & Inflação $0 \%$ & Inflação 2\% & Inflação $4,5 \%$ & Inflação 7\% & Inflação $10 \%$ \\
\hline Regressão & $\mathrm{W}(0,05)$ & $\mathrm{W}(0,07)$ & $\mathrm{W}(0,095)$ & $\mathrm{W}(0,12)$ & $\mathrm{W}(0,15)$ \\
\hline OLS estático (Tabela 2) & 0,3351 & 0,4067 & 0,4849 & 0,5546 & 0,6306 \\
\hline OLS dinâmico, $\mathrm{p}=1$ (Tabela 4) & 0,4970 & 0,5887 & 0,6864 & 0,7719 & 0,8635 \\
\hline OLS dinâmico, $\mathrm{p}=2$ (Tabela 4) & 0,5250 & 0,6201 & 0,7211 & 0,8093 & 0,9035 \\
\hline OLS dinâmico, $\mathrm{p}=3$ (Tabela 4 ) & 0,5531 & 0,6515 & 0,7557 & 0,8465 & 0,9434 \\
\hline \multicolumn{6}{|c|}{ Painel C } \\
\hline & Inflação $0 \%$ & Inflação 2\% & Inflação $4,5 \%$ & Inflação 7\% & Inflação $10 \%$ \\
\hline Regressão & $\mathrm{W}(0,07)$ & $\mathrm{W}(0,09)$ & $\mathrm{W}(0,115)$ & $\mathrm{W}(0,14)$ & $\mathrm{W}(0,17)$ \\
\hline OLS estático (Tabela 2) & 0,4067 & 0,4700 & 0,5412 & 0,6060 & 0,6776 \\
\hline OLS dinâmico, $\mathrm{p}=1$ (Tabela 4) & 0,5887 & 0,6680 & 0,7556 & 0,8341 & 0,9196 \\
\hline OLS dinâmico, $\mathrm{p}=2$ (Tabela 4$)$ & 0,6201 & 0,7021 & 0,7924 & 0,8733 & 0,9611 \\
\hline OLS dinâmico, $\mathrm{p}=3$ (Tabela 4) & 0,6515 & 0,7361 & 0,8292 & 0,9123 & 1,0025 \\
\hline \multicolumn{6}{|c|}{ Painel D } \\
\hline & Inflação $0 \%$ & Inflação $2 \%$ & Inflação $4,5 \%$ & Inflação 7\% & Inflação $10 \%$ \\
\hline Regressão & $\mathrm{W}(0,09)$ & $\mathrm{W}(0,11)$ & $\mathrm{W}(0,135)$ & $\mathrm{W}(0,16)$ & $\mathrm{W}(0,19)$ \\
\hline OLS estático (Tabela 2) & 0,4700 & 0,5276 & 0,5935 & 0,6544 & 0,7223 \\
\hline OLS dinâmico, $\mathrm{p}=1$ (Tabela 4) & 0,6680 & 0,7389 & 0,8190 & 0,8920 & 0,9724 \\
\hline OLS dinâmico, $\mathrm{p}=2$ (Tabela 4) & 0,7021 & 0,7752 & 0,8577 & 0,9328 & 1,0153 \\
\hline OLS dinâmico, $\mathrm{p}=3$ (Tabela 4) & 0,7361 & 0,8115 & 0,8964 & 0,9735 & 1,0581 \\
\hline
\end{tabular}

Fonte: Valores estimados pelos autores.

Obs.: Os custos de bem-estar da inflação calculados assumem taxas real de juros de estado estacionário

equivalentes à: i) Painel $A, r=0,03$; ii) Painel $B, r=0,05$; iii) Painel $C, r=0,07$; e Painel $D, r=0,09$, sob

pressuposto de inflação nula ou estabilidade de preços. 


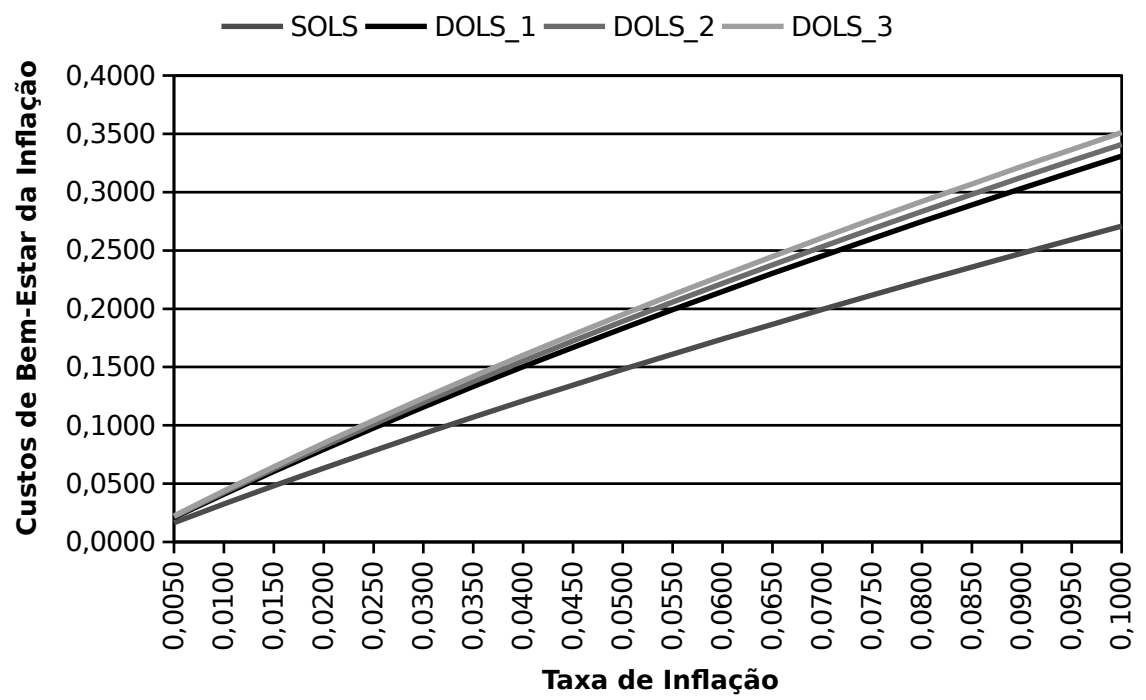

Fonte: Elaboração própria.

Figura 2: Custos de Bem-Estar da Inflação com aproximação de Simonsen \& Cysne

a taxa de deflação. A complexidade de tal operação pode ser posta diante da literatura em que não se observa tal tentativa prática.

\section{Considerações Finais}

Neste estudo, discutiram-se estruturas funcionais log-log e semi-log associadas às medidas de custo social da inflação, tomando por base os trabalhos elaborados por Lucas (2000) e por Ireland (2009). A contribuição adicional foi, também, apresentar resultados por meio tanto das aproximações de Bailey quanto da aproximação de Simonsen e Cysne.

Os testes empíricos, para um período de dezessete anos, resultaram na corroboração da forma funcional log-log proposta para a curva de demanda por moeda brasileira, permitindo em seguida estimar os custos de bem-estar da inflação pós-Plano Real. Em outras palavras, a perspectiva do ambiente inflacionário não teria afetado a relação de demanda por moeda apresentada em Simonsen \& Cysne (2007) para o período anterior ao Plano Real, uma vez que os resultados ficaram próximos.

Ao se considerar o modelo dinâmico restrito, os resultados evidenciaram que a elasticidade-juros foi maior em valor absoluto, sugerindo que a inserção de leads e lags tornam a demanda por moeda pouco mais sensível influenciando, consequentemente, um pouco mais as medidas de custo social. Um resultado importante, mas não surpreendente, é a clara sensibilidade dos custos de bem-estar da inflação quando se busca uma redução da inflação de $10 \%$ para algo próximo aos patamares de países desenvolvidos. Ainda, observamse decrescentes custos de bem-estar da inflação quando se considera taxa real de juros anual de estado estacionário crescente, indicando efeitos negativos de se manter essa última taxa a níveis maiores dentro da economia brasileira. Ou seja, os ganhos de bem-estar da redução da inflação são relativamente menores em maiores níveis de taxa real de juros anual de estado estacionário. 
Por exemplo, usando a aproximação de Simonsen e Cysne, para uma inflação média de aproximadamente $4,5 \%$ ao ano, o custo de bem-estar estaria entre 0,15 e $0,20 \%$ da renda, considerando uma taxa real de juros anual de estado estacionário de $5 \%$ ao ano. Se a taxa real neutra é de $7 \%$ a.a., os custos seriam algo entre 0,13 e $0,18 \%$ da renda.

Conforme Simonsen \& Cysne (2007), para uma inflação média em torno de $96.9 \%$ ao ano entre 1947 e 1993, o Brasil teve um custo de bem-estar ao redor de $2.93 \%$ da renda. Tal resultado comparado ao do presente trabalho, em que a inflação média foi de $7,5 \%$ a.a. dentro do período de estudo, demonstra um significativo ganho de bem-estar com o Plano Real. Todavia, os resultados apresentados neste estudo, de uma forma geral, apontam que apesar desse ganho, a política brasileira deve não só buscar reduzir e estabilizar os preços aos níveis de seus parceiros comerciais desenvolvidos, mas também diminuir a taxa real de juros anual de estado estacionário para níveis de países desenvolvidos.

\section{Agradecimentos}

Os autores agradecem ao Conselho de Desenvolvimento Científico e Tecnológico (CNPq) pelo apoio financeiro por meio do edital MCTI /CNPq / MEC/CAPES No $07 / 2011$.

\section{Referências Bibliográficas}

Anderson, R. G. \& Rasche, R. H. (2001), The remarkable stability of monetary base velocity in the united states, 1919-1999, Working paper 2001-008a, Federal Reserve Bank of St. Louis.

Arnosti, M. (2003), Demanda de moeda no brasil: Uma análise por meio do filtro de kalman, Master's thesis, Dissertação de Mestrado, Universidade Federal do Rio Grande do Sul - UFRGS.

Bailey, M. J. (1956), 'The welfare cost of inflationary finance', Journal of Political Economy 64(2), 93-110.

Barbosa, F. (1978), 'A demanda de moeda no brasil: resenha da evidência empírica', Pesquisa e Planejamento Econômico 8(1), 33-82.

Barbosa, F. (1993), 'Hiperinflação e a forma funcional da equação de demanda de moeda', Análise Econômica 11(20), 5-16.

Barbosa, F., Pereira, P. \& Sallum, E. (1995), 'A substituição de moeda no brasil: a moeda indexada', Pesquisa e Planejamento Econômico 25(3), 407-426.

Caetano, S. M., Souza Júnior, J. R. C. \& Moura, G. V. (2012), Estimativas da função de reação do banco central do brasil e da taxa de juros neutra no período de 2003 a 2011.

Cagan, P. (1956), The Monetary Dynamics of Hyperinflation, Chicago: University of Chicago Press.

Canêdo-Pinheiro, M. (2011), 'Modelo de cagan e quebras estruturais: evidências para o brasil (1970-94)', Economia Aplicada 15(2), 151-176. 
Cardoso, E. (1981), 'Uma equação para a demanda de moeda no brasil', Pesquisa e Planejamento Econômico 11(3), 617-636.

Contador, C. (1974), 'Desenvolvimento financeiro, liquidez, e substituição ente ativos no brasil: a experiência recente', Pesquisa e Planejamento Econômico 4(2), 245-284.

Cysne, R. (1985), 'Moeda indexada', Revista Brasileira de Economia 39(1), 5774.

Cysne, R. (2003), 'Divisia index, inflation, and welfare', Journal of Money, Credit, and Banking 35(2), 221-238.

Cysne, R. (2005), 'A general-equilibrium closed-form solution to the welfare costs of inflation', Revista Brasileira de Economia 59(2), 209-214.

Cysne, R. (2009), 'Bailey's measure of welfare costs of inflation as a general equilibrium measure', Journal of Money, Credit, and Banking 41(2), 451-459.

Cysne, R. \& Turchick, D. (2010), 'Welfare costs of inflation when interestbearing deposits are disregarded: A calculation of the bias', Journal of Economic, Dynamics E Control 34, 1015-1030.

Dias, M. (2000), A revision on the welfare costs of inflation and seignorage in brazil: A model with leisure, Uem-pme working paper.

Friedman, M. (1969), The Optimum Quantity of Money, Chicago: Aldine Publishing Company.

Gregory, A. W. \& Hansen, B. E. (1996), 'Residual-based tests for cointegration in models with regime shifts', Journal of Econometrics 70, 99-126.

Hamilton, J. D. (1994), Time Series Analysis, Princeton: Princeton University Press.

Harberger, A. C. (1971), 'Three basic postulates for applied welfare economics: An interpretation', Journal of Economic Literature 9(3), 785-797.

IBGE (2007), Apresentação da nova série do sistema de contas nacionais, referencia 2000, Technical report, Rio de Janeiro: IBGE (Nota Metodológica n. 1).

URL: Disponivel em: <http://www.ibge.gov.br/home/estatistica/indicadores/pib/pdf/01_apresentacao.pdf> Acessado em: 07/01/2009

Ireland, P. N. (2009), 'On the welfare cost of inflation and the recent behavior of money demand', American Economic Review 99(3), 1040-1052.

Lucas, R. E., J. (2000), 'Inflation and welfare', Econometrica 68(2), 247-74.

Meltzer, A. H. (1963), 'The demand for money: The evidence from the time series', Journal of Political Economy 71(3), 219-46.

Nakane, M. (1994), Testes de exogeneidade fraca e a superexogeneidade para a demanda por moeda no brasil, Technical report, Rio de Janeiro: $18^{\circ}$ Prêmio BNDES de Economia. 
Newey, W. K. \& West, K. D. (1987), 'A simple, positive semi-definite, heteroskedasticity and autocorrelation consistent covariance matrix', Econometrica 55(3), 703-08.

Pastore, A. C. (1969), 'Inflação e política monetária no brasil', Revista Brasileira de Economia 23(1), 92-123.

Pastore, A. C. (1995), Senhoriagem e inflação: O caso brasileiro.

Phillips, P. C. B. \& Perron, P. (1998), 'Testing for a unit root in time series regression', Biometrika 75(2), 335-46.

Prado, E. (1978), 'Demanda de moeda no brasil: o problema da forma funcional', Pesquisa e Planejamento Econômico 8(3), 781-794.

Romer, D. (2005), Advanced Macroeconomics, 3 edn, New York: McGraw Hill.

Rossi, J. (1988), 'A demanda por moeda no brasil: o que ocorreu a partir de 1980?', Pesquisa e Planejamento Econômico 18(1), 37-54.

Rossi, J. (1994), 'O modelo hiperinflacionário de demanda por moeda de cagan e o caso do brasil', Pesquisa e Planejamento Econômico 24(1), 73-96.

Rossi, J. (2008), 'O custo de bem-estar da inflação: Cálculo tentativo com o uso de um modelo de equilíbrio geral', Estudos Econômicos 38(1), 127-150.

Serletis, A. \& Yavari, K. (2004), 'The welfare cost of inflation in canada and the united states', Economics Letters 84, 199-204.

Silveira, A. M. (1973), 'The demand for money: The evidence from brazilian economy', Journal of Money, Credit, and Banking 5(1), 113-140.

Simonsen, M. \& Cysne, R. (2001), 'Welfare costs of inflation and interestbearing money', Journal of Money, Credit, and Banking 33(1), 90-100.

Simonsen, M. \& Cysne, R. (2007), Macroeconomia, 3 edn, São Paulo: Atlas.

Stock, J. H. \& Watson, M. W. (1993), 'A simple estimator of cointegrating vectors in higher order integrated systems', Econometrica 61(4), 783-820.

Tourinho, O. (1995), 'A demanda por moeda em processos de inflação elevada', Pesquisa e Planejamento Econômico 25(1), 7-68.

Tourinho, O. (1996), A demanda por moeda no brasil: 1974/95, Technical Report 419, Texto para Discussão do IPEA.

Triches, D. (1992), Demanda por moeda no brasil e a causalidade entre as variáveis monetárias e a taxa de inflação 1972/87, Technical report, Rio de Janeiro: $16^{\circ}$ Prêmio de Economia BNDES.

Yavari, K. \& Serletis, A. (2011), 'Inflation and welfare in latin america', Open Economics Review 22, 39-52.

Yoshino, J. (2002), The brazilian welfare costs of inflation, Technical report, FEA-USP Working Paper.

Zivot, E. \& Andrews, D. W. K. (1992), 'Further evidence on the great crash, the oil-price shock, and the unit-root hypothesis', Journal of Business E Economic Statistics 10(3), 251-270. 